\title{
NIVEL DE SATISFAÇÃO COM O ATENDIMENTO EM FISIOTERAPIA MEDIDO PELO MEDRISK
}

\section{LEVEL SATISFACTION WITH PHYSIOTHERAPY SERVICE MEASURED BY MEDRISK}

\author{
Maria Natália Cardoso \\ Elisa Brosina de Leon ${ }^{2}$
}

\begin{abstract}
Resumo
Avaliar a qualidade é informação útil para tomada de decisões e melhoria dos serviços. Este artigo pretende apresentar o nível de satisfação e o bem-estar das usuárias de um serviço de Fisioterapia Pélvica. O questionário MedRisk Instrument for Mensuring Patient Satisfaction (MRPS) foi aplicado em idosas $(n=30)$ para mensurar a satisfação. Quanto maior a pontuação no MRPS (máximo 5), maior a satisfação do usuário com o serviço. Os resultados demonstram uma média de $4,81 \pm 0,38$ pontos após a realização das sessões de fisioterapia pélvica nos itens avaliados. Conclui-se que o serviço de fisioterapia pélvica possui alto grau de satisfação percebidos pelas clientes e que os itens relacionados a interação entre fisioterapeuta e paciente tiveram uma influência maior na satisfação geral.
\end{abstract}

Palavras-chave: Avaliação da qualidade dos cuidados de saúde; fisioterapia; satisfação do usuário.

\begin{abstract}
Assessing quality is useful information for decision making and service improvement. This article intends to present the level of satisfaction and wellbeing of users of a Pelvic Physiotherapy service. The MedRisk Instrument for Mesuring Patient Satisfaction (MRPS) questionnaire was applied to elderly women $(n=30)$ to measure satisfaction. The higher the MRPS score (maximum 5), the greater the user's satisfaction with the service. The results show an average of $4.81 \pm 0.38$ points after performing the pelvic physiotherapy sessions on the evaluated items. It is concluded that the pelvic physiotherapy service has a high degree of satisfaction perceived by clients and that items related to the interaction between physiotherapist and patient had a greater influence on overall satisfaction.
\end{abstract}

Key-words: Quality Assurance, health care; therapy, physical; satisfaction, consumer.

\footnotetext{
${ }^{1}$ Docente do curso de Fisioterapia da Faculdade de Educação Física e Fisioterapia da Universidade Federal do Amazonas. E-mail: nataliacardoso301@gmail.com

${ }^{2}$ Doutora em Biotecnologia pela Universidade Federal do Amazonas, Brasil. Professora Adjunto IV da Universidade Federal do Amazonas, Brasil. Agencias de Fomento: Conselho Nacional de Desenvolvimento Científico e Tecnológico (CNPQ); Coordenação de Aperfeiçoamento de Pessoal de Nível Superior (CAPES); Universidade Federal do Amazonas (UFAM); Fundação Universidade Aberta da Terceira Idade (FUNATI). E-mail: elisadleon@ufam.edu.br
}

Artigo recebido em: 26 de agosto de 2021. Artigo aceito em 17 de novembro de 2021. 


\section{Introdução}

O conceito de qualidade para Parasuraman, Berry e Zeithaml (1985) é a diferença entre as expectativas (esperado) dos clientes sobre o serviço a receber e suas percepções (percebido) sobre os serviços efetivamente entregues(MIGUEL; SOUSA; FREIRE, 2017). Portanto, avaliar os níveis de qualidade, constitui informação útil para a tomada de decisões estratégicas e operacionais, capazes de contribuir para uma subsequente melhoria da qualidade dos serviços prestados (TINOCO; CÂNDIDO; FELICIANO, 2017).

A avaliação da qualidade é muito importante para qualquer tipo de instituição, pois é ela que determina se os resultados da implementação de ações de produção de qualidade conferem confiabilidade e capacidade ao plano de gestão da empresa prestadora de serviço, permitindo que a avaliação sirva como elemento sinalizador das ações estratégicas das organizações (SADOYAMA et al., 2019). O enfoque na qualidade evolui à medida que as relações sociais e econômicas do homem se tornam mais complexas. Mais do que uma simples evolução do conceito de Qualidade em Serviços, implica num processo contínuo e recorrente com um aumento progressivo da sua importância na economia mundial, tornando-se um fator preponderante (MIGUEL; SOUSA; FREIRE, 2017).

A qualidade na prestação de serviços é um diferencial e depende de um conjunto de envolvidos no processo para satisfazer as necessidades do cliente ou usurário. Os serviços são baseados em pessoas e equipamentos, mas é o componente humano que prevalece, por esta razão é muito difícil uniformizá-los e padronizá-los. Neste sentido, saber a quem é direcionado o serviço, ou seja, conhecer quem são os clientes, é importante para que as empresas definam exatamente como, onde e porquê deve-se ser prestado o serviço, conhecer os clientes é uma vantagem competitiva significativa para uma empresa de serviços (NOGUEIRA MEDEIROS et al., 2020).

Nas últimas décadas, a qualidade dos serviços de saúde tem ganhado magnitude, passando por constantes construções conceituais e metodológicas. Definir níveis de qualidade em saúde exige grande complexidade dada à peculiaridade das instituições, pois estas possuem padrões de qualidade cada vez mais elevados, o que as fazem buscar meios para mensurar seus serviços (SANTOS; SARDINHA; SANTOS, 2017). Nota-se assim que a avaliação da satisfação do paciente acerca da assistência que está sendo 
oferecida é um importante item de avaliação no que diz respeito à qualidade do atendimento, uma vez que, um usuário satisfeito demonstra maior adesão ao tratamento, continuando a utilizar os serviços (ESPIRIDIÃO; TRAD, 2005; SOUZA; GRIEBELER; GODOY, 2007).

Essa avaliação faz parte dos estudos das relações interpessoais no âmbito dos serviços de saúde, fortalecendo a participação da comunidade nos processos de planejamento e avaliação. Ademais, o protagonismo dos usuários nesse processo tornaos avaliadores reais, amplifica seus direitos como cidadãos e os envolvem também na corresponsabilização da produção do cuidado à saúde, podendo influenciar no modo da oferta dos cuidados e possibilitar uma maior qualidade e resolutividade (PROTASIO et al., 2017).

A satisfação do atendimento na saúde tem natureza multidimensional, ou seja, o paciente pode estar satisfeito com um ou mais aspectos da assistência à saúde e, simultaneamente, estar insatisfeito com outros aspectos. Portanto a satisfação está sempre relacionada às expectativas do paciente, podendo ser alterada conforme as expectativas e comparativos do paciente mudam (OLIVEIRA et al., 2010). A mesma sido utilizada como um indicador de qualidade da atenção aos cuidados recebidos no setor da saúde (MEDEIROS et al., 2016).

Esse é um complexo atributo e é estabelecido de acordo com as expectativas do usuário. Logo, pacientes satisfeitos são mais propensos a ter lealdade com os provedores de tratamento e colaborar com o regime estabelecido (ARIMA; WIECEK; MATSUSHIMA, 2006). A fisioterapia apresenta uma série de características que influenciam a satisfação do paciente: a interação com o paciente sempre leva mais tempo do que uma consulta médica, a terapia envolve maior contato físico, geralmente exige a participação ativa do paciente e a terapia pode causar dor e ser encarada como uma ameaça física (SUDA; UEMURA; VELASCO, 2012).

$\mathrm{Na}$ fisioterapia pélvica esse contato é ainda mais íntimo, pois é trabalhado um conjunto muscular pertencente a uma área do corpo que atualmente ainda é um tabu para a maioria das mulheres: o assoalho pélvico (MEDEIROS et al., 2016). Uma avaliação de caráter qualitativo e a relação do nível de satisfação com o tipo e tempo de patologia se faz necessária, a fim de se entender melhor a percepção do paciente atendido e a relação dessa percepção com o nível de satisfação apresentado (SUDA; UEMURA; VELASCO, 2012). 
Assim, este estudo teve como objetivo apontar o nível de satisfação das pacientes do sexo feminino com idade igual ou superior a 60 anos que utilizam o serviço de Fisioterapia Pélvica para incontinência urinária mista, de urgência e de esforço na Universidade Aberta da Terceira Idade aplicando a versão brasileira do questionário MedRisk Instrument for Mensuring Patient Satisfaction (MRPS).

\section{Procedimentos Metodológicos}

Este estudo trata-se de um estudo transversal composto por mulheres com 60 anos ou mais com indicação para tratamento conservador da Incontinência Urinária no Serviço de Fisioterapia Pélvica da Fundação da Universidade Aberta da Terceira Idade, localizado na cidade de Manaus-Am. Composto por 30 pacientes, atendidas no serviço de Fisioterapia Pélvica na fundação da Universidade Aberta da Terceira Idade, ManausAm, no período entre Setembro de 2018 a Novembro de 2019. Foram utilizados como critérios de inclusão: mulheres, com idade igual ou superior a 60 anos, que utilizaram o serviço de Fisioterapia Pélvica da Universidade Aberta da Terceira Idade. Foram utilizados como critérios de exclusão: pacientes que não completaram as sessões fisioterapêuticas prescritas e pacientes que apresentaram número de faltas não justificadas superior a 3 sessões.

\section{Instrumentos de pesquisa}

As características gerais das participantes foram coletadas por meio de uma ficha de avaliação que apresenta questões referentes aos dados demográficos e antropométricos, além de informações sobre o quadro clínico das participantes, como a condição de saúde e os sintomas da paciente.

Para mensuração do nível de satisfação das pacientes pós atendimento fisioterapêutico foi aplicado o MedRisk, Instrument for Mensuring Patient Satisfaction (MRPS) (MEDEIROS et al., 2016). O MRPS é um questionário de 13 itens, subdividido em 13 fatores, desenvolvido especificamente para a Fisioterapia. O instrumento é dividido em fatores: Fator 1, denominado interpessoal, contém seis itens relacionados à interação terapeuta-paciente ou relacionado à interação do paciente com os outros funcionários da clínica. $\mathrm{O}$ fator 2 , denominado eficiência e conveniência, é composto por 3 itens, como a afirmativa "Os horários de atendimento dessa clínica foram convenientes para mim”. O Fator 3, denominado educação do paciente, possui dois itens 
referentes ao comprometimento do fisioterapeuta em conscientizar e educar seus pacientes, como a afirmativa "Meu fisioterapeuta forneceu-me instruções detalhadas sobre o programa de exercícios para casa". Finalmente, o instrumento possui dois itens não alocados em fatores específicos, que são considerados itens globais, como a afirmativa "Eu retornaria a essa clínica para futuros serviços". O paciente responde seu nível de satisfação para cada item por meio de uma escala do tipo Likert, que varia de 1 ("discordo completamente") a 5 ("concordo completamente"), além da opção "não se aplica", disponível para algumas afirmativas. Escores mais altos representam maior satisfação. Esse instrumento foi previamente traduzido e transculturalmente adaptado para a população brasileira.

Também foi aplicada a Escala de Percepção do Efeito Global (Global Perceived Effect - GPE) A GPE verifica a impressão global de recuperação atual do paciente, comparando o início de seus sintomas com sua condição de saúde atual. E uma escala do tipo Likert de 9 pontos, variando de 1 ("extremamente melhor") a 9 ("extremamente pior"), em que pontuações mais baixas representam pacientes que estão melhor e pontuações maiores representam aqueles que pioraram após o início do tratamento em comparação com o início dos seus sintomas.

\section{Procedimento de coleta de dados}

Os potenciais participantes foram abordados no serviço de Fisioterapia Pélvica no dia da alta, ao fim da reavaliação. Todas as pacientes receberam informação sobre o objetivo e o procedimento do estudo, em seguida foram respondidos os questionários MRPS, GPE. O estudo foi aprovado pelo Comitê de Ética em Pesquisa da UFAM no 3.670.863. Todas as participantes assinaram o Termo de Consentimento Livre e Esclarecido, que garante o anonimato e a confidencialidade das respostas. Não houve a discriminação na seleção dos indivíduos, nem exposição a riscos desnecessários.

\section{Análise de dados}

Para análise de dados foram calculadas as médias (desvios-padrão - DP) das variáveis contínuas, e a descrição dos dados categóricos foi calculada por meio de números (porcentagens). Para calcular a satisfação dos pacientes foram calculadas médias (DP) para cada item do MRPS e para o escore total do questionário. 


\section{Resultados}

A tabela 1 mostra as características físicas das pacientes estudadas quanto a idade, índice de massa corporal (IMC) e quanto ao número de gestações, partos normais e cesárias, onde a média de gestações dessas 30 pacientes resultou em 3,96 $\pm 2,78$. Houve um número maior de partos normais 2,64 $\pm 2,60$ comparado ao cesáreo $0,75 \pm 0,84$ evento esse que influencia diretamente no aumento da probabilidade de adquirir incontinência urinária. Outro fator que tem grande peso para o surgimento da Incontinência Urinária é a Episiotomia, nesse estudo 10 pacientes relataram ter passado por esse procedimento.

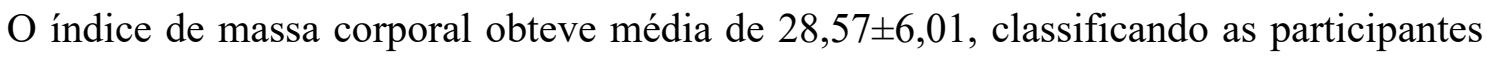
como acima do peso. O total de 30 pacientes, que receberam o serviço de Fisioterapia Pélvica para o tratamento de Incontinência Urinária, obtiveram alta e responderam ao questionário do MedRisk. A média de idade foi 60,86 $\pm 8,63$.

Tabela 1 - Características físicas das participantes.

\begin{tabular}{lc}
\hline \multicolumn{1}{c}{ Variável } & $(\mathbf{n}=\mathbf{3 0})$ \\
\hline Idade (média $\pm \mathrm{dp})$ & $60,86( \pm 8,63)$ \\
Índice de Massa Corporal $(\mathrm{IMC})($ média $\pm \mathrm{dp})$ & $28,57( \pm 6,01)$ \\
Número de Gestações (média $\pm \mathrm{dp})$ & $3,96( \pm 2,78)$ \\
Partos Normais (média $\pm \mathrm{dp})$ & $2,64( \pm 2,60)$ \\
Cesárias (média \pm dp) & $0,75( \pm 0,84)$ \\
Tipo de incontinência & \\
Incontinência Urinária de Emergência (IUE) (n; \%) & $56,66 \%$ \\
Incontinência Urinária de Urgência (IUU) & $6,66 \%$ \\
Incontinência Urinária Mista (IUM) & $23,33 \%$ \\
Tempo médio do aparecimento de sintomas & 45,4 \\
Número médio de atendimento & 13,11 \\
\hline
\end{tabular}

Em referência ao tempo de sintomas da incontinência urinária a média foi de 45,4 meses. A situação dos sintomas 16 pacientes informaram terem piorado desde o surgimento deles até o dia em que haviam procurado o serviço, apenas 2 disseram ter melhorado, 10 afirmaram permanecer do mesmo jeito e 2 não responderam.

Com relação ao estado civil: 6 afirmaram ser solteiras; 14 casadas; 3 divorciadas e 5 eram viúvas. Com referência à queixa principal: 17 pacientes relataram possuir Incontinência Urinária de Emergência (IUE); 7 pacientes Incontinência Urinária Mista (IUM); 2 pacientes Incontinência Urinária de Urgência (IUU); 2 Bexiga Hiperativa 
(BH) e 2 não responderam. Ainda com relação a tabela 1 é possível analisar as características das pacientes estudadas referente à incontinência urinária e o tipo de incontinência, em porcentagem (\%). É possível observar que das 30 pacientes $56,66 \%$ foram diagnosticadas com Incontinência Urinária de Emergência onde há a perca de urina ao subir uma escada, carregar pesos, levantar da cadeira, fazer exercícios como corrida e agachamento. A de menor incidência foi a Incontinência Urinária de Urgência, que é a dificuldade de segurar a urina e ocorrente perca dela a caminho do banheiro, com 6,66\%. E a segunda de maior incidência foi a Incontinência Urinária Mista com 23,33\%, que é quando há o diagnóstico dos dois tipos anteriores de Incontinência Urinária.

O MedRisk foi aplicado após a alta das pacientes. Quando perguntadas sobre o tempo que levavam para se deslocar de sua casa para a Policlínica: 7 (23,33\%) disseram levar menos de 15 minutos; 9 (30\%) relataram levar entre 16 e 30 minutos; 5 (16,66\%) entre 31 e 60 minutos; $4(13,33 \%)$ afirmaram levar mais de 60 minutos e $5(16,66 \%)$ não responderam.

A descrição dos resultados estatísticos dos itens globais e dos dois itens individuais estão descritos na tabela 2 , em média e desvio padrão.

A menor média foi relacionada ao conforto na sala de espera (iluminação, temperatura, móveis) no entanto quando analisamos a resposta das 30 participantes, porém, mesmo nesse item, $100 \%$ apresentou avaliação entre concordaram completamente e concordam. Essa discrepância pode ter ocorrido pelo tamanho da sala, variações de temperatura da sala por ser uma sala de uso coletivo. O item que se referia ao retorno à clínica para futuros serviços ou tratamentos foi o que obteve média mais alta $(4,93 \pm 0,25)$ com $86 \%$ das participantes respondendo concordo completamente. A maior parte dos itens individuas obtiveram médias acima de 4,8 com a exceção da média $(4,75 \pm 0,43)$ do item onde questiona-se se o fisioterapeuta respondeu a todas as questões do paciente, assim sendo $96 \%$ avaliaram entre concordo e concordo completamente, a média pode ter sofrido uma queda pelo fato de uma paciente não ter respondido à questão. As perguntas globais são direcionadas ao ambiente e aos funcionários, neste âmbito a afirmativa relacionada a limpeza da clínica e suas

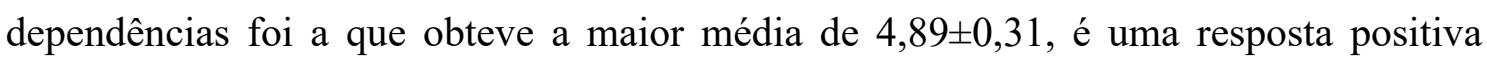
salientando a importância que os usuários, dos serviços de saúde, dão ao quesito higienização do ambiente ao mesmo tempo que nos expõe a sua ótica do meio de 
maneira geral. O último item denota maior importância, haja vista que se relaciona diretamente ao tratamento no momento em que é dada a oportunidade do paciente avaliar, de forma clara e direta, o atendimento do fisioterapeuta nos expondo, através da sua percepção, à aplicabilidade das condutas terapêuticas utilizadas e à eficácia do tratamento, comprovando, em conjunto, a eficiência do serviço ofertado. A pergunta que rege essas conclusões indaga ao paciente como está sua atual condição comparada como ele estava antes de começar o serviço fisioterapêtico, para dar a resposta o usuário pode marcar entre os números de 1 (extremamente melhor) a 9 (extremamente pior). Das 30 pacientes que participaram do estudo, 66\% marcaram estar extremamente melhor e $33 \%$ muito melhor, resultando em uma média de $1,34 \pm 0,48$.

Tabela 2 - Pontuação média dos 13 itens avaliados (11 globais e 2 individuais)

\begin{tabular}{|c|c|c|c|c|}
\hline Itens & Mínimo & Média & DP & Máximo \\
\hline 1 - A recepcionista foi cortês? & 4 & 4,79 & $\pm 0,41$ & 5 \\
\hline 2 - O processo de registro foi adequado? & 4 & 4,75 & $\pm 0,43$ & 5 \\
\hline 3 - A sala de espera era confortável? (Iluminação, temperatura, móveis). & 4 & 4,68 & $\pm 0,47$ & 5 \\
\hline $\begin{array}{l}4 \text { - Os horários de atendimento dessa clínica foram convenientes para } \\
\text { mim. }\end{array}$ & 3 & 4,72 & $\pm 0,52$ & 5 \\
\hline $\begin{array}{l}5 \text { - Meu fisioterapeuta me explicou cuidadosamente os tratamentos que } \\
\text { eu recebi. }\end{array}$ & 4 & 4,82 & $\pm 0,38$ & 5 \\
\hline 6 - Meu fisioterapeuta me tratou respeitosamente. & 4 & 4,89 & $\pm 0,30$ & 5 \\
\hline 7 - Os funcionários da clínica me trataram respeitosamente. & 4 & 4,82 & $\pm 0,38$ & 5 \\
\hline 8 - Meu fisioterapeuta respondeu a todas as minhas questões. & 4 & 4,75 & $\pm 0,43$ & 5 \\
\hline $\begin{array}{l}9 \text { - Meu fisioterapeuta aconselhou-me sobre a forma de evitar futuras } \\
\text { complicações. }\end{array}$ & 4 & 4,89 & $\pm 0,30$ & 5 \\
\hline 10 - A clínica e suas dependências estavam limpas. & 4 & 4,89 & $\pm 0,31$ & 5 \\
\hline $\begin{array}{l}11 \text { - Meu fisioterapeuta forneceu-me instruções detalhadas sobre meu } \\
\text { programa de exercícios para casa. }\end{array}$ & 4 & 4,82 & $\pm 0,38$ & 5 \\
\hline $\begin{array}{l}12 \text { - De forma geral estou completamente satisfeito (a) com os serviços } \\
\text { que recebi do meu fisioterapeuta. }\end{array}$ & 4 & 4,89 & $\pm 0,30$ & 5 \\
\hline 13 - Eu retornaria a esta clínica para futuros serviços ou tratamentos. & 4 & 4,93 & $\pm 0,25$ & 5 \\
\hline $\begin{array}{l}14 \text { - Como está a sua atual condição comparada como você estava antes } \\
\text { de começar o serviço fisioterápico? }\end{array}$ & 2 & 1,34 & $\pm 0,48$ & 1 \\
\hline
\end{tabular}

Quando questionadas se a recepcionista foi cortês: 7 (23,33\%) relataram concordar e $21(70 \%)$ concordaram completamente. Em resposta a terceira questão se o processo de registro foi adequado: 10 (33,33\%) afirmaram concordar e 19 (63,33\%) disseram concordar completamente. Sobre a sala de espera, se era confortável: 9 (30\%) concordaram e $18(60 \%)$ concordaram completamente. Se os horários de atendimento 
da clínica eram confortáveis: $7(23,33 \%)$ concordaram e 21 (70\%) concordaram completamente. Quando questionadas se o fisioterapeuta as tratou cordialmente: 4 $(13,33 \%)$ afirmaram concordar e $25(83,33 \%)$ concordaram completamente. Se os funcionários da clínica a trataram cordialmente: 6 (20\%) disseram concordar e 23 $(76,66 \%)$ concordaram completamente. Se o fisioterapeuta respondeu a todas as suas questões: $8(26,66 \%)$ concordaram e $21(70 \%)$ concordaram completamente. Se o fisioterapeuta as aconselhou como evitar futuros problemas: $4(13,33 \%)$ disseram concordar e $25(83,33 \%)$ concordaram completamente. Se a clínica e suas dependências estavam limpas: $4(13,33 \%)$ concordaram e 24 (80\%) concordaram completamente. Se o fisioterapeuta forneceu informações detalhadas sobre os exercícios em casa: 6 (20\%) concordaram e $23(76,66 \%)$ concordaram completamente. Quando questionadas se estão completamente satisfeitas com o serviço fornecido pelo fisioterapeuta: 4 (13,33\%) disseram concordar e $25(83,33 \%)$ concordaram completamente. Se retornariam a clínica para futuros serviços: $3(10 \%)$ concordaram e $26(86,66 \%)$ concordaram completamente. Quando questionada sobre sua atual condição comparada como estava antes de iniciar o tratamento fisioterapêutico: 20 (66,66\%) relataram estarem extremamente melhores e $10(33,33 \%)$ muito melhor.

\section{Discussão}

A qualidade dos serviços está relacionada à habilidade de minimizar as discrepâncias entre as expectativas e percepções de clientes e seus fornecedores. Segundo Zeithaml (1988, p. 3), "um serviço é de qualidade somente quando iguala ou supera as expectativas que o cliente tem a respeito dele”. Portanto, a avaliação da qualidade do serviço deve ser feita comparando as expectativas de execução com a percepção a respeito do serviço recebido. Esse é o parâmetro que melhor demonstra a satisfação, ou insatisfação, do cliente e, portanto, a qualidade do serviço oferecido. Essa avaliação da satisfação deve ser feita de forma ativa, pelo questionamento e pela comunicação com os clientes(LEITE; FILHO, 2007).

O modelo conceitual de medição da qualidade do serviço, desenvolvido por Zeithaml et al. (1985), parte do conceito de que a qualidade do serviço se determina pela comparação do serviço esperado com o recebido. Ela é geralmente avaliada com base em cinco dimensões da qualidade do serviço, que são: confiabilidade, capacidade de resposta, segurança, elementos tangíveis e empatia. $\mathrm{O}$ instrumento utilizado para 
avaliação da qualidade do serviço consiste em um questionário específico para o serviço que se pretende medir. Nesse questionário, refletem-se os atributos para cada dimensão da qualidade do serviço (confiabilidade, garantia etc.) (LEITE; FILHO, 2007).

A satisfação do consumidor é um apanhado de acertos ocorridos no ambiente de serviços que envolve, acima de tudo, conhecer e exceder as necessidades e demandas deste consumidor. Nesse contexto, a satisfação dos consumidores não é estática, possui dinamismo. Tal fato eleva a importância de acompanhamento constante dos níveis de satisfação de maneira adequada, ou seja, com a utilização de instrumentos que possuam alto grau de confiabilidade e, acima de tudo, de fácil interpretação (MOYANO; ANGNES; CORREAA, 2020).

A avaliação de serviços de saúde é área de conhecimento e de prática que possibilita oferecer diretrizes e opções para o processo de planejamento, podendo favorecer o controle técnico e social dos serviços prestados à sociedade (MISHIMA et al., 2010). Nesse contexto, a satisfação do usuário representa um potente indicador de resultado para avaliação dos serviços de saúde, considerando ser fundamental conhecer como os usuários avaliam o atendimento a eles prestado para repensar as práticas profissionais e sobre a forma de organização dos serviços (GOMIDE et al., 2018).

Quanto maior a pontuação encontrada, maior a satisfação do usuário com o serviço, sendo o maior valor possível igual a 5 (DE FÁTIMA COSTA OLIVEIRA et al., 2014). Assim podemos dizer que o grau de satisfação encontrado nesse estudo foi elevado, com média de 4,81. Em síntese avaliar a satisfação do paciente acerca da assistência que está sendo oferecida é um importante item de avaliação no que diz respeito à qualidade do atendimento, uma vez que, um usuário satisfeito demonstra maior adesão ao tratamento, continuando a utilizar os serviços (CORDEIRO DE MEDEIROS et al., 2016). Ademais a satisfação do atendimento na saúde tem natureza multidimensional, ou seja, o paciente pode estar satisfeito com um ou mais aspectos da assistência à saúde e, simultaneamente, estar insatisfeito com outros aspectos. Portanto a satisfação está sempre relacionada às expectativas do paciente, podendo ser alterada conforme as expectativas e comparativos do paciente mudam (BORGES et al., 2003).

Os dados encontrados nesta pesquisa estão de acordo com os encontrados em outros estudos, demonstrando que os itens com maior satisfação estão relacionados à interação terapeuta-paciente e ao respeito nela envolvido. E esses itens mostraram maior correlação com a satisfação geral (DE FÁTIMA COSTA OLIVEIRA et al., 2014). Logo 
a satisfação do paciente é diretamente relacionada com a interação pacientefisioterapeuta, assim, fatores como: explicações claras sobre o tratamento e terapeutas que demonstram habilidades de comunicação bem desenvolvidas seriam mais importantes para a satisfação do paciente do que a localização da clínica ou a qualidade dos equipamentos disponíveis (HINGARAJIA, 2013).

Assim, a capacidade do terapeuta em manter a comunicação e expor sobre o tratamento está entre os itens mais importantes por parte dos usuários que expressam sua satisfação com os atributos e a atenção que lhes é oferecida, uma vez que se sentem favorecidos pelo tratamento recebido. Uma das estratégias para aprimorar a atenção oferecida aos pacientes é analisar a satisfação dos mesmos, sendo de extrema importância e servindo como feedback para o profissional da saúde aprimorar seus atendimentos. Deste modo, as pesquisas são realizadas reunindo informações que ajudam a incorporar as opiniões dos pacientes na prática clínica e, assim, aprimorar o tratamento prescrito aos mesmos (SANTANA, 2016).

O presente estudo mostrou que o MedRisk é de fácil aplicação e interpretação além de promover o aumento da satisfação e a identificação de item específicos que mais influenciam na percepção geral da satisfação do usuário do serviço de físioterapia, dando a oportunidade de o serviço estar sempre evoluindo e melhorando com o intuito de ter um tratamento cada vez mais eficaz e que motive o paciente a buscar sua melhora. Assim como ajudar a compreender porque o paciente certas vezes decide abandonar o serviço e interromper o tratamento, o que não é possível com os demais instrumentos desenvolvidos até então. Além disso, por possuir questões gerais acerca do tratamento fisioterapêutico e do ambiente, o MedRisk acaba por se tornar um questionário amplo podendo ser utilizado em diversas áreas da Fisioterapia, tal como presente em estudos da ortopedia, pacientes hospitalizados e o atual estudo na Fisioterapia Pélvica.

\section{Limitações}

O estudo realizado apresentou limitações quanto à sua população relacionado ao fato da espera pela alta das pacientes, que ocorria após 10 atendimentos, no mínimo.

Ocasionando outra limitação importante referente ao tamanho da amostra, apresentando um número reduzido que permite considerar os resultados encontrados apenas para a população em questão. 


\section{Conclusão}

Podemos concluir com este trabalho que o serviço prestado no serviço de fisioterapia pélvica apresentou alto grau de satisfação pelas suas usuárias e que os itens relacionados a interação entre fisioterapeuta e paciente tiveram uma influência maior na satisfação geral.

\section{Agradecimentos}

Agradecimentos à Fundação Universidade Aberta da Terceira Idade (FUnATI) por disponibilizar as instalação para realização do Projeto Vida Livre. As acadêmicas do Curso de graduação em Fisioterapia: Rosana Queiroz, Lunna Barroso, Diulliany Nunes, Jaqueline Frota, Debora Lorena, Giovanna Águila e Fisioterapeuta Maga Rocha pela participação no Projeto. Agradecimentos pelo fomento financeiro à Universidade Federal do Amazonas e ao Conselho Nacional de Desenvolvimento Científico e Tecnológico (CNPq).

\section{Referências Bibliográficas}

ARIMA, A. A.; WIECEK, D. P.; MATSUSHIMA, L. C. Universidade Estadual Paulista "Júlio de Mesquita Filho " Faculdade de Medicina de Botucatu. p. 1-20, 2006.

BORGES, R. et al. Incontinência urinária no idoso. Acta Cirúrgica Brasileira, v. 18, n. Supl 5, p. 47-51, 2003.

CORDEIRO DE MEDEIROS, F. et al. Satisfação de pacientes que recebem cuidados fisioterapêuticos para condições musculoesqueléticas: um estudo transversal Satisfaction of patients receiving physiotherapy care for musculoskeletal conditions: a cross-sectional study Satisfacción de paciente. Fisioter pesq., n. 23(1), p. 105-110, 2016.

DE FÁTIMA COSTA OLIVEIRA, N. et al. Measurement properties of the Brazilian Portuguese version of the medrisk instrument for measuring patient satisfaction with physical therapy care. Journal of Orthopaedic and Sports Physical Therapy, v. 44, n. 11, p. 879-889, 2014.

ESPIRIDIÃO, M.; TRAD, L. A. B. Avaliação da satisfação de usuários. Ciência \& saúde coletiva, v. 10, n. sup, p. 303-312, 2005.

GOMIDE, M. F. S. et al. A satisfação do usuário com a atenção primária á saúde: uma análise do acesso e acolhimento. Interface: Communication, Health, Education, v. 22 , n. 65 , p. $387-398,2018$.

HINGARAJIA, D. N. a Psychometric Analysis of Patient Satisfaction With Physical Therapy Care. Innovative Journal of Medical and Health Science, v. 3, p. 219-223, 
2013.

LEITE, R. S.; FILHO, C. G. U U Um Estudo Empírico Da Aplicação Do Índice Europeu De Satisfação De Clientes (Ecsi) No Brasil an Empirical Study of European Customer Satisfaction Index (Ecsi) Application in Brazil. Revista de Administração Mackenzie, v. 8, n. 4, p. 178-200, 2007.

MEDEIROS, F. C. DE et al. Satisfação de pacientes que recebem cuidados fisioterapêuticos para condições musculoesqueléticas: um estudo transversal. Fisioterapia e Pesquisa, v. 23, n. 1, p. 105-110, 2016.

MIGUEL, M. C.; SOUSA, M. M. DE; FREIRE, V. F. Avaliação da qualidade nos espaços de popularização da ciência e tecnologia com ênfase na astronomia e nos ambientes imersivos de tecnologias da informação e comunicação . Revista de iniciação científica, tecnológica e artística, v. 6, p. 64-76, 2017.

MISHIMA, S. M. et al. Artigo Original A assistência na saúde da família sob a perspectiva dos usuários ${ }^{1}$. Latino-Am. Enfermagem, v. 18, n. 3, 2010.

MOYANO, C. A. M.; ANGNES, D. L.; CORRÊA, M. D. Satisfação dos clientes bancários: uma análise bibliométrica dos artigos acadêmicos publicados na última década. Revista Brasileira de Administração Científica, v. 11, n. 2, p. 24-41, 2020.

NOGUEIRA MEDEIROS, M. et al. Application of the SERVQUAL Model in the evaluation of the quality of the service provided by a security company in Higher Education Institution. Revista Gestão da Produção Operações e Sistemas, v. 15, n. 2, p. 01-22, 2020.

OLIVEIRA, E. et al. Avaliação dos fatores relacionados à ocorrência da incontinência urinária feminina. Revista da Associação Médica Brasileira, v. 56(6), n. November, p. 688-90, 2010.

PROTASIO, A. P. L. et al. Satisfação do usuário da atenção básica em saúde por regiões do Brasil: $1^{\circ}$ ciclo de avaliação externa do PMAQ-AB. Ciencia e Saude Coletiva, v. 22, n. 6, p. 1829-1844, 2017.

SADOYAMA, G. et al. Avaliação da qualidade de serviços de equipe interdisciplinar nos processos educa vos em diabetes Revista EDaPECI INTRODUÇÃO O Diabetes Mellitus ( DM ) é um relevante e crescente problema de saúde pública, devido elevada incidência , prevalência , morbim. Revista EDaPECi, v. 18, p. 19-31, 2019.

SANTANA, L. A. Avaliação do nível de satisfação dos pacientes atendidos no núcleo de fisioterapia e reabilitação física de Sobradinho/DF. Fisioterapia Brasil, v. 16, n. 1, p. $38,2016$.

SANTOS, M. A. DOS; SARDINHA, A. H. DE L.; SANTOS, L. N. DOS. Satisfação dos usuários com os cuidados dos enfermeiros. Revista gaucha de enfermagem, v. 38, n. 1, p. e57506, 2017.

SOUZA, A. M.; GRIEBELER, D.; GODOY, L. P. Qualidade na prestação de serviços fisioterápicos. Produção, v. 17, n. 3, p. 435-453, 2007.

SUDA, E. Y.; UEMURA, M. D.; VELASCO, E. Avaliação da satisfação dos pacientes atendidos em uma clínica-escola de fisioterapia de Santo André, SP. Fisioterapia e Pesquisa, v. 16, n. 2, p. 126-131, 2012.

TINOCO, P. C. R.; CÂNDIDO, C. J. F.; FELICIANO, I. M. P. L. The importance of 
perceived quality assessment in radiology and the impact of sociodemographic factors in a Portuguese public hospital. Portuguese Journal of Public Health, v. 35, n. 3, 2017. 